\title{
Cauda Equina Syndrome: A Review of 15 Patients Who Underwent Percutaneous Transforaminal Endoscopic Lumbar Discectomy (PTELD) Under Local Anaesthesia
}

\author{
Krishnan A, MS, Kohli R, MS, Degulmadi D, MS, Mayi S, MS, Ranjan R, MS, Dave B, MS \\ Department of Spine Surgery, Stavya Spine Hospital and Research Institute, Ahmedabad, India
}

This is an open-access article distributed under the terms of the Creative Commons Attribution License, which permits unrestricted use,
distribution, and reproduction in any medium, provided the original work is properly cited
Date of submission: 27 th December 2019
Date of acceptance: 19 th April 2020

\section{ABSTRACT}

Introduction: To analyse the results of Cauda Equina Syndrome (CES) operated by Percutaneous Transforaminal Endoscopic Lumbar Discectomy (PTELD).

Materials and Methods: The study is a retrospective series of 15 patients operated by PTELD. Bladder dysfunction was classified as incomplete CES (CESI) and complete CES retention (CESR). Bladder / motor recovery rate and its timing, Oswestry Disability Index (ODI), Visual Analogue Score (VAS), patient satisfaction index, and sexual dysfunction were used to measure the outcome objectively. Additionally, in CESR patients, post-void residual (PVR) urine was measured by sonography. Complications and technical problems were noted.

Results: There were ten patients of CESI and five patients of CESR. The average follow-up was 20.33(12.05) months. Bladder symptoms recovery was $100 \%$, and motor recovery was $80 \%$. VAS for back pain recovered to $0.53(0.52)$ from 8(2.39). VAS for leg pain recovered to $0.13(0.35)$ from 9.20(1.32). ODI improved to 6.07(2.85) from 77.52(13.20). The time to the recovery of bladder function was $1.47(1.55)$ days. All CESR patient's abnormal PVR urine was normalised at five weeks post-operative. No complications were reported. However, five technical executional problems occurred.

Conclusion: PTELD can be considered for CES treatment due to its substantial and quick recovery advantages. However, more evidence support is needed to make it a practice recommendation.

\section{Keywords:}

cauda equina syndrome, transforaminal endoscopy, percutaneous, minimal invasive spine surgery

\section{INTRODUCTION}

Cauda equina syndrome (CES) is one of the most serious and complicated spinal pathologies ${ }^{1,2}$. It has been reported as the extreme presentation of 1-3\% of Lumbar Disc Herniation (LDH) patients ${ }^{2-4}$. Conventionally, treatment for CES is open laminectomy/discectomy (MED: Microscopic Decompression) $)^{5}$. Although minimally invasive approaches have been reported, they are often not recommended due to perceived manipulations required in a smaller approach. Additionally, there are high chances of residual disabilities of limb and/or bladder and/or sexual dysfunction leading to unhappiness and medico-legal litigations ${ }^{3,6}$.

The use of Percutaneous Transforaminal Endoscopic Lumbar Discectomy (PTELD) for treating CES is sparsely mentioned in the literature ${ }^{7-13}$. Indications of PTELD have evolved significantly and in India, the catalyst of the evolution was the mentorship of Dr Gore ${ }^{11,14}$. The advantages of PTELD are the use of local anaesthesia, day-care, least pain, speedy recovery, preservation of muscles, stability, low blood loss, and minimal post-operative complications ${ }^{8-10,13,14}$.

There are other added advantages of PTELD in CES that are worth considering. There is less manipulation of compromised neural tissues, and urgent surgery is possible without lengthy pre-anaesthetic preparations ${ }^{8,9,13,14,15,16}$. There are also the significant considerations of pain reduction and enhanced recovery with minimally invasive surgeries ${ }^{17}$. Recently, few articles on PTELD in CES have been published that quote unbelievably excellent outcomes, the majority of which reveal surprisingly quick and complete recoveries $^{8-12}$. We report our retrospective non-consecutive case series and experience of PTELD in CES due to LDH. 


\section{MATERIALS AND METHODS}

This study was a retrospective analysis of all operated PTELD cases. Informed consent was taken in all cases, which included the option of open surgery if optimal decompression fails. The study was conducted from January 2014 to December 2018. Inclusion criteria were a single level LDH with one or more of the following red flags: (1) bladder and/or bowel dysfunction, (2) reduced sensation in the saddle area, (3) sexual dysfunction with a possible neurologic deficit in the lower limb (motor/sensory loss, reflex change) ${ }^{1,4}$.

Hospital medical records and image databases were analysed. Patient demographics reviewed included age, gender, back and/or leg complaint (acute, sub-acute or chronic), onset/duration of bladder problems, and neurological status. MRC (Medical Research Council) grading scale of 0 to 5 was used in assessing motor power. A significant weakness score was assigned if the muscle power was less than grade three.

CES classification: CES was categorised into two types. The first was CESI (incomplete), in which patients had a spectrum of urinary difficulties such as hesitancy, straining, low-pressure stream, and/or increased frequency. The second was CESR (with retention) ${ }^{6}$. Ultrasonographic measurement of post-void residual (PVR) urine was done in all CESR patients with measurement of more than $200 \mathrm{ml}$ considered positive ${ }^{18}$. Additionally, the bladder dysfunction of CES presentation was classified as either within 24 hours or more than 24 hours.

LDH Presentation acuteness: Patient presentation of LDH was classified as acute (within 48 hours), sub-acute (within one month), or chronic. Chronicity was labelled when the patient came in with a progressive lower back pain (LBP) or radiculopathy of more than three months duration. The onset of bladder dysfunction signaled the start of CES.

MRI (Magnetic Resonance Imaging) scans were assessed for the presence of a complete myelo-block. Overt instability was ruled out and the radiological type of intra-canalicular LDH was labelled according to the axial view (central or para-central) and sagittal view (for migration). Lateral recess stenosis (LCS), if present, was noted. Calcifications (CC) of disc/annulus or end plate spurs were noted and confirmed in intra-operative findings.

Surgical techniques: An independent anaesthesiologist was present during the whole surgical procedure. The surgery was done with local anaesthesia (LA) and conscious sedation. The patient was put on protected prone position and received supplemental oxygen. Forty-five minutes before surgery, intramuscular midazolam $(0.05 \mathrm{mg} / \mathrm{kg})$ and diclofenac were given. Half a dose of titrated infusion of dexmedetomidine $(0.5-1 \mathrm{mcg} / \mathrm{kg})$ was given slowly with an intravenous dose of $1 \mathrm{mcg} / \mathrm{kg}$ of fentanyl bolus ten minutes before the surgery. This was followed by additional doses as needed.

A uni-portal approach was used. An imaginary line was drawn to the annular puncture site and the skin site was marked for the surgical trajectory planned. The angle was between $0^{\circ}$ and $30^{\circ}$, and the puncture point was $12-16 \mathrm{~cm}$ from midline depending on the basic technique used. The intended needle entry tract was infiltrated with $1 \%$ lidocaine plus bupivacaine at 1:1 ratio. A 16-gauge needle was inserted fluoroscopically in the Kambin's triangle with continuous patient's feedback and $1.5 \mathrm{ml}$ further infiltration on the annulus. A $7 \mathrm{~mm}$ incision was made and followed with tapered dilating trocar. The beveled working cannula was railroaded, and then through that the endoscope was introduced. The removal of the offending compression was done with any of the inventory as needed [Carl Storz-Gore System-Germany / Maxmore system-Germany or Richard wolf system-Germany]. Prolapsed disc excision was done along with compressive ventral tissues. The techniques employed depended upon the pathology and were standard basic techniques of outside in (OI) or inside out (IO) or FEE (flat epidural entry), with modifications as needed.

Inside Out (IO): An approach at a 15 to $20^{\circ}$ angle was taken. The disc was pierced, and the sub annular disc was removed before cutting the annular anchorage and working in epidural space $^{13,14}$.

Outside In (OI): Blind non-visualised reamed foraminoplasty using Maxmore Tom Shidi reamers was done to reach epidural space directly ${ }^{15}$.

FEE (flat epidural entry): The flat approach was taken nearly at an angle of $0^{\circ}$ landing in the epidural space avoiding the piercing of the disc and facet reaming ${ }^{16}$.

An endoscopic visualised BF (burred foraminoplasty) or OI approach was taken when there was a technical requirement to reach more dorsal. Nouvag system [Goldache, Switzerland] was used. When CC was contributing to the ventral stenosis endoscopic osteotomes and burr were used (CVD: Calcified Ventral Decompression). In bilaterally symptomatic cases, if the adequacy of decompression was not satisfactory from index uni-portal approach (UPA) due to limited reach to opposite ventral side, then in the same stage, opposite side transforaminal approach was taken (Bi-lateral, Bi-portal approach; BLBP). The decompression endpoints were by complete visualisation of the roots, dural sac, probing, dural pulsations, irrigation flutter, and cough impulse.

Sub-cuticular stitches were taken, and all patients were sent for MRI. All patients were mobilised as per their tolerance and limb power. Requisite patients were advised to undertake passive/active physiotherapy. The urinary catheter 
was not done pre-operatively for any patient except the ones needing GA. The level of surgery, operating time (OR Time), and estimated blood loss (EBL) were noted. All patients were reviewed based on the time between the onset of CES to surgery, the length of hospital stay (LHS), and the months of follow-up. The evaluation of neurological and functional outcomes was done using validated measures.

ODI: In ODI, nine components (out of ten) were used, and sexual dysfunction was not used. Total added score as a percentage is expressed. Ten-point Visual Analog Scale score (VAS) scale for LBP and leg pain was used.

Motor and Bladder Improvement: Improvement beyond MRC grade three was considered as recovery. The bladder outcome (recovered or not) was assessed. Bladder status was noted as recovered to normal, recovered to CESI status, or remaining as CESR with the time (days) to the recovery.

Sexual dysfunction scoring: Sexual dysfunction scoring on the basis of the SHIM (Sexual Health Inventory in Males) and a non-validated questionnaire to assess female sexual dysfunction was collected as a follow-up only. The SHIM is a five-item questionnaire validated as a screening tool for erectile dysfunction ${ }^{6,19}$. However, in conservative societies like India, a woman is expected to maintain silence when confronted with issues of her own sexuality. So, instead of available validated female sexual dysfunction scores, we used a non-validated self-assessment questionnaire. The females were asked to give an overall score to categorise their sexual function as good, fair, or poor considering factors of frequency, satisfaction, dryness, and pain ${ }^{6}$.

Overall satisfaction: Patient Satisfaction Index was used as a self-assessment tool to determine the overall satisfaction outcome with 3 parameters - one highly satisfied, two moderately satisfied, and three not satisfied ${ }^{6}$. The quality of residual LBP was categorised into NILBP (non-instability LBP) and ILBP (instability-related LBP). If LBP was present, as a constant backache not related to workload and not aggravated by loading, which felt more like a pulling or stiffness in the back, it was labeled as NILBP. The pain in ILBP was typically associated with positional change, such as standing up from sitting, bending forward, and floor activities, or related to workload aggravation ${ }^{6}$. Complications and technical problems were noted. The following recovery rates were assessed:

Bladder recovery rate $=[$ Number of patients with complete bladder recovery/Number of patients with pre-operative bladder dysfunction] * $100 \%$

Motor recovery rate $=[$ Number of patients with motor recovery (MRC $>$ grade 3)/Number of patients with preoperative motor weakness (MRC $<$ grade 3$)] * 100 \%$
Statistics: Patient demographics and characteristic categorical variables were analysed, and the mean (standard deviation) for all applicable variables were calculated. Each category was compared by using appropriate statistical tools such as the Pearson correlations, unpaired Student t-test, and paired t-tests. Statistical analysis was performed with IBM SPSS software ver. 20.0 [IBM Corp, Armonk, NY, USA]. A p-value of $<0.05$ was considered to be statistically significant.

\section{RESULTS}

A total of 15 non-consecutive patients (13 males and 2 females) were operated for CES by the author (AK). None needed a conversion to open surgery intra-operatively or later. Their demographic presentation variables were summarised (Table I).

The age of patients was 38.5 (15.6) years. Acute onset was present in three patients. The symptom duration was 76.1 (53.2) days. The duration of onset of bladder dysfunction was 50 (35.3) hours. And, four out of fifteen patients presented themselves within 24 hours. LBP was present in 14; tingling numbness was present in all 15, bilateral affection in ten, motor weakness in ten, saddle affection in seven and CESR in five patients, respectively. PVR urine 264ml (43.9) was present in all five CESR patients.

All patients had LDH. The most common level of lesion in our study was L4-5 ( $\mathrm{n}=10)$. Additional LCS was present in one patient. All the patients had complete myelographic block. None of the patients had any instability. Twelve patients had central LDH. Three had CC, which was annulus calcification in one and endplate spurs in two. Seven patients had additional co-morbidities of obesity, hypertension, and/or diabetes mellitus.

All patients, except for one who demanded general anaesthesia (GA), were operated under LA. All the patients received surgery within 24 hours of presentation. However, only four patients presented within 24 hours of the onset of bladder dysfunction. No midnight emergency surgery was done. Surgery was done using the basic techniques of IO $(n=11)$, OI $(n=02)$, and FEE $(n=02)$, respectively. Both OI was needed in $15 \mathrm{~S} 1 \mathrm{LDH}$. In three patients with $\mathrm{CC}$, the "inside-out" technique needed additional technique modifications (CVD) for removal of the hardened tissue. Additionally, $\mathrm{BF}$ was needed in four $(\mathrm{IO}=3, \mathrm{OI}=1)$ patients for improving the reach. Two patients needed BLBP for complete decompression. Post-operatively in all patients, MRI confirmed the decompression. The average EBL was 31.3 (9.2) $\mathrm{ml}$ and duration of surgery 84.3 (22.3) minutes, respectively. All patients were immediately mobilised. 
The average follow-up period was of 20.3 (12.1) months. The pre-operative back pain VAS was 8 (2.4) and it reduced to $0.5(0.5)$ during the final follow-up. The pre-operative leg pain VAS was 9.2 (1.3), and it nearly vanished postoperatively and was reduced to $0.13(0.4)$ at the time of the final follow-up. The results across these variables were statistically significant (Table II).

All CESI patients had complete bladder recovery immediately $(100 \%)$. Of the CESR patients, three did not have symptoms of bladder dysfunction at discharge. Two patients had some straining difficulty, which lasted for a week in one patient and 48 hours in the other. First of these patients had to undergo indwelling catheterisation postoperatively that was removed after 72 hours. This was the same patient who was operated under GA. PVR urine on the follow-up of all CESR patients at five weeks was nil. The time to the recovery of bladder function was 1.5 (1.6) days.

The bladder recovery rate was $100 \%$, and the motor recovery rate was $80 \%$. Significant muscle power recovered in the remaining two patients to grade four MRC (one tibialis anterior with triceps surae and other triceps surae only). But these two had weakness in the toes present, though improved from previous ' 0 ' MRC grade to grade 2 . Out of the ten patients with a substantial deficit, eight recovered. There was non-bothering tingling numbness in legs present in five patients at the final follow-up. Residual saddle affection remained in two patients, and five completely recovered.

The pre-operative ODI was 77.5 (13.2), and it improved quickly and progressively to 6.1 (2.9) at the final follow-up (Fig. 1). The patient satisfaction index was one in all patients. No NILBP was noted in any patient. ILBP was present in nine patients, which were occasional and were noted less frequently in those who followed lifestyle modification advice.

At follow-up, according to the SHIM scale, three patients had moderate erectile dysfunction. All of these three patients told that it was pre-existing. Female sexual function was good in both the patients.

There were no major complications except technical problems of retrieving and decompressing in two patients who needed BLBP. One patient had more bleeding than usual. Two patients had fogging issues of the endoscope and needed to change to a second endoscope. One patient, after improvement of CES completely, at four weeks developed an episode of back pain (VAS 5). His activities were not affected, and a repeat MRI showed a recurrent small sequestrated disc extrusion. He became non-symptomatic again in three weeks through conservative means. One patient needed re-catheterisation and developed a urinary tract infection, which was conservatively treated, and eventually improved. One patient of CESR came back after
10 days with a mild LBP increase and was immediately subjected to MRI because of our anxiety despite no physical deterioration (Fig. 2).

\section{DISCUSSION}

CES was first reported in 1934 by Mixter and Barr, who pioneered it via a transdural approach ${ }^{20}$. Individuals in their fourth and fifth decades of life are prone to LDH and progression to $\mathrm{CES}^{2,21}$. Our sample set for the study was mostly in their thirties, and men were more susceptible to be affected $(n=13,86.7 \%)$ than women. Various classification systems of CES have been reported without uniformity but bladder dysfunction is considered universal with rare sparing aswell ${ }^{4,22,23,24}$. All our patients $(n=15,100 \%)$ depicted classic symptoms of $\mathrm{CES}^{4}$.

MRI is the investigation of choice in $\mathrm{CES}^{25}$. All our patients $(100 \%)$ had complete myelo-block, which in itself suggests severe compression. Small window surgeries are not recommended in CES and wider conventional opening may precipitate an instability ${ }^{6,26,27}$. Manipulation is dreaded and one report of spinal surgery itself as the cause of approximately $15 \%$ of the total numbers of CES brings to notice the true under-reporting in literature ${ }^{28}$. Trans-dural technique to avoid retraction has also been reported ${ }^{1,20}$. One case of PIELD (Interlaminar Endoscopy) in the largest series of CES $(n=9)$ also developed new transient motor weakness $^{10}$. Moreover, for the treatment of upper LDH, PTELD has been more recommended ${ }^{29,30}$. In our series, we had three cases of upper $\mathrm{LDH}$, and they both showed complete recovery.

The recovery in CES may take months to years, as reported conventionally ${ }^{3,31}$. A three-case report of anterior lumbar surgery and another report of endoscope assisted OLIF (Oblique lumbar Interbody Fusion) in CES, report a dramatic quick recovery in bladder function ${ }^{32,33}$. Although these are relatively open surgery, this should raise a prudent question of an underestimated manipulative injury in posterior surgeries. The excellent outcome of bladder recovery in our series may be because the ventral access surgery could be executed at the earliest possible instance.

Post-surgical pain is the biggest concern before most surgeries. It invades and erodes wellbeing and increases anxiety-stress of the patient ${ }^{17}$. Moreover, loss of sexual function and significant physical disability also looms heavily on CES patients ${ }^{1-4}$. The recovery of bladder function happened within 48 hours in all but one patient in our study. The minimal pain after PTELD would be a positive factor towards a faster recovery. Comparatively, in conventional surgeries, only $50-70 \%$ of patients get either a true recovery or compensatory balanced outcome of bladder function ${ }^{3}$. 

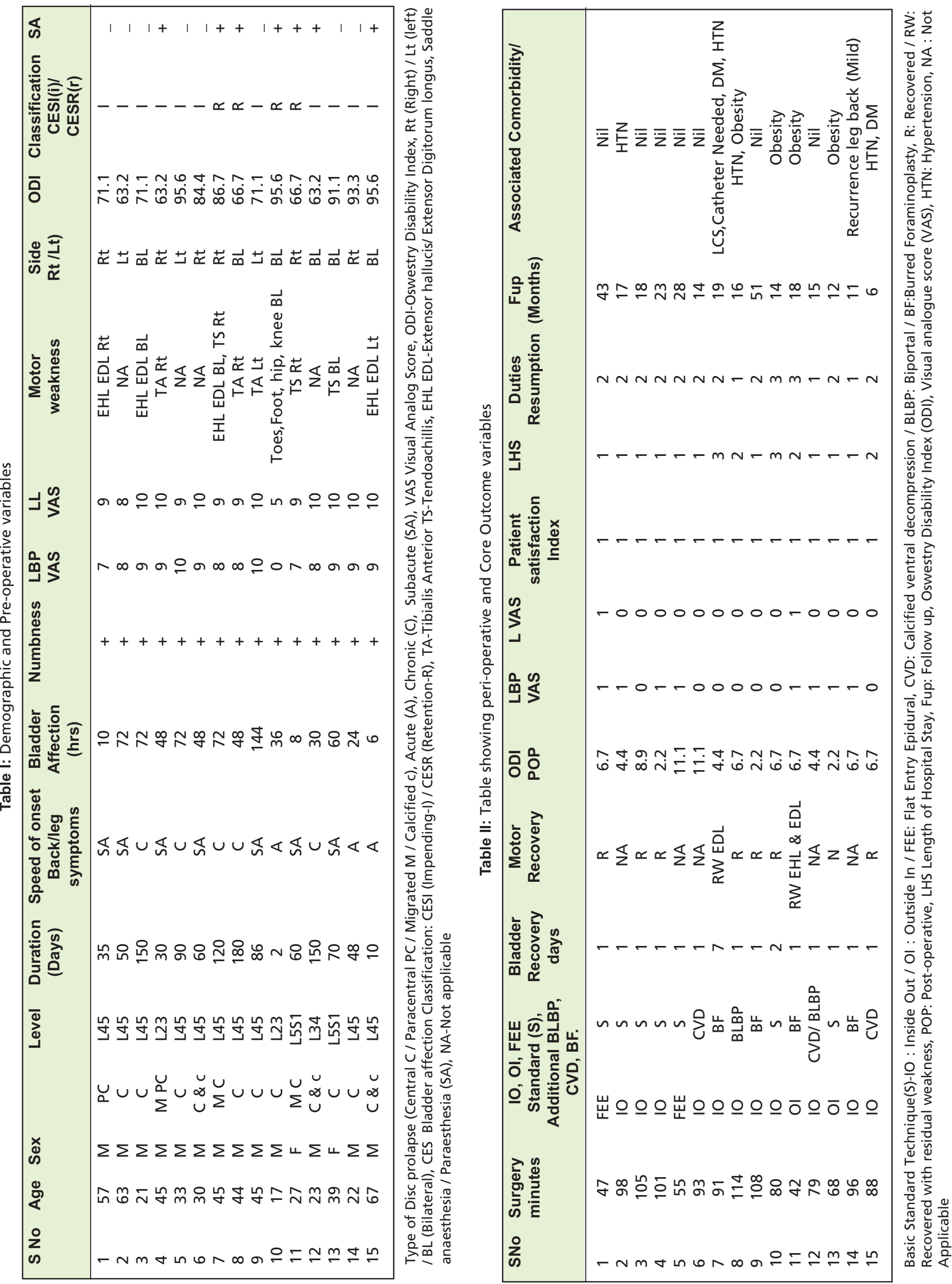


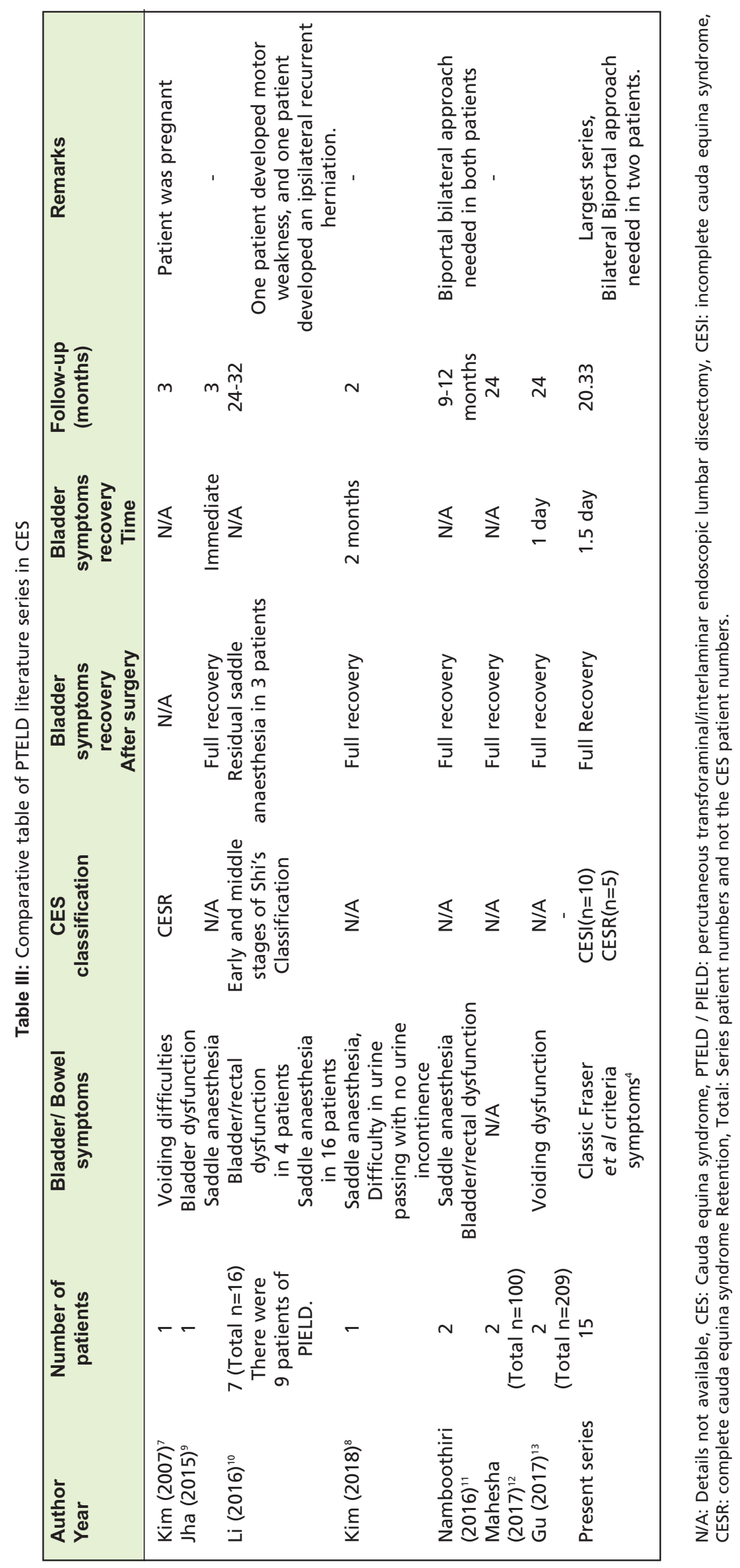


$\mathrm{P}<.001$

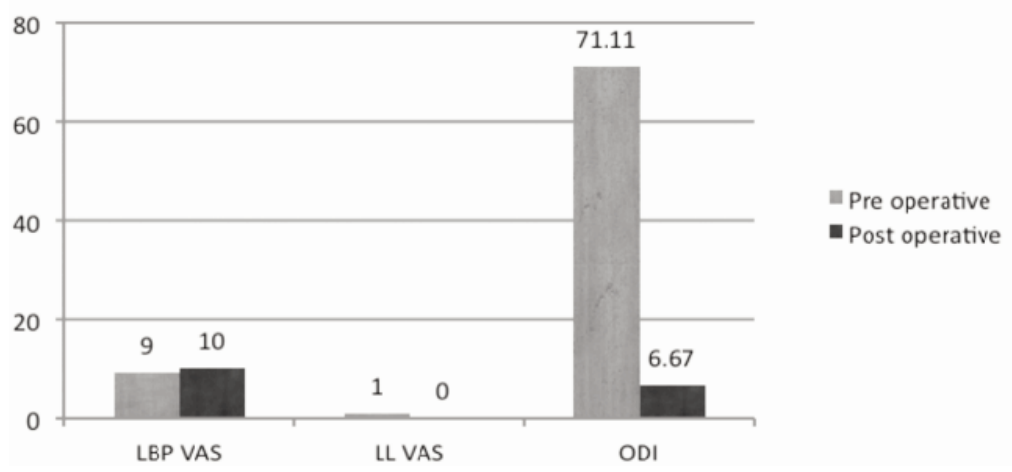

Fig. 1: Bar diagram showing outcome parameters, Low back pain VAS, Limb VAS and ODI score.
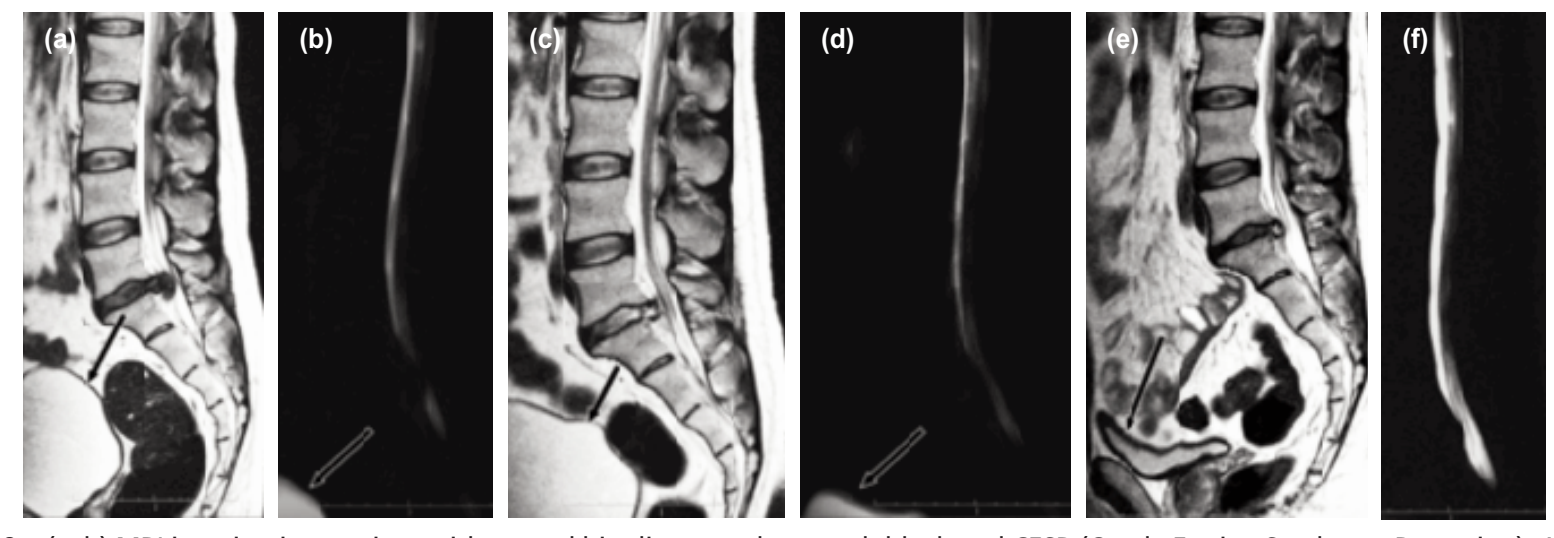

Fig. 2: ( $a$, b) MRI imaging in a patient with central big disc, complete myeloblock and CESR (Cauda Equina Syndrome Retention). Arrow showing distended bladder in MRI and myelogram. (c, d) Immediate post-operatively, patient had slow urine stream and needed straining to empty. The MRI showing a complete decompression and a clear myelogram. Arrow showing distended bladder postoperatively in MRI and myelogram as well. $(\mathrm{e}, \mathrm{f}) \mathrm{MRI}$ and myelogram on follow-up ten days shows complete empty bladder (arrow) without any clinical bladder dysfunction.
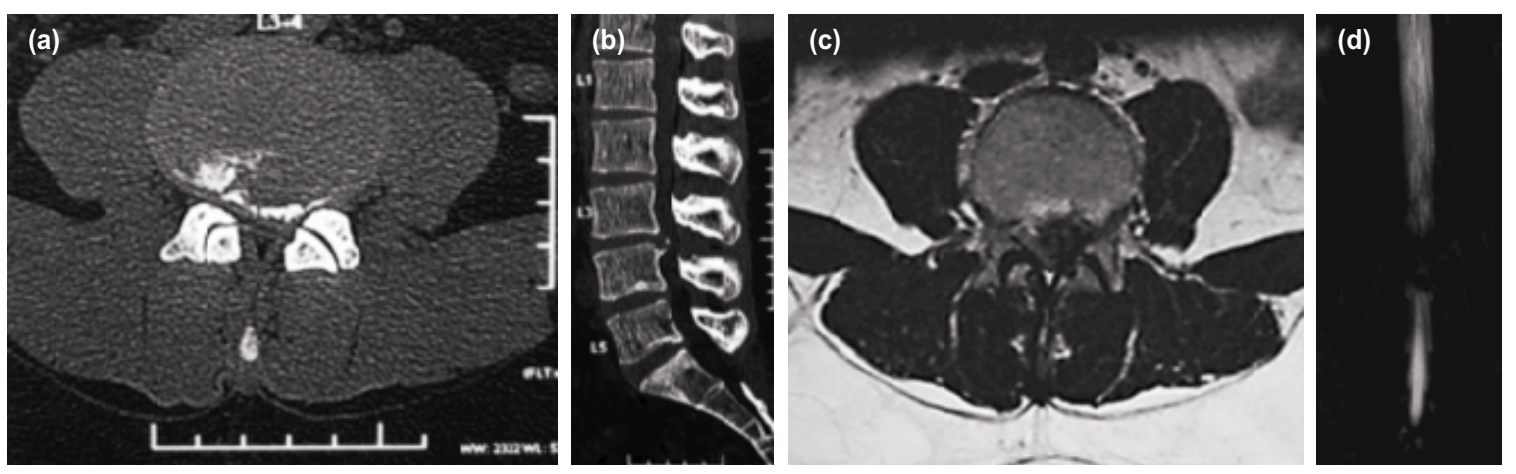

Fig. 3: (a) Pre-operative CT (Computed Tomography) scan axial (b) and sagittal, (c) axial MRI in a 23-year-old male patient of CES with bilateral leg affection and vesicular dysfunction showing a calcified central LDH (Lumbar Disc Herniation). (d) MRI myelogram showing complete block. 

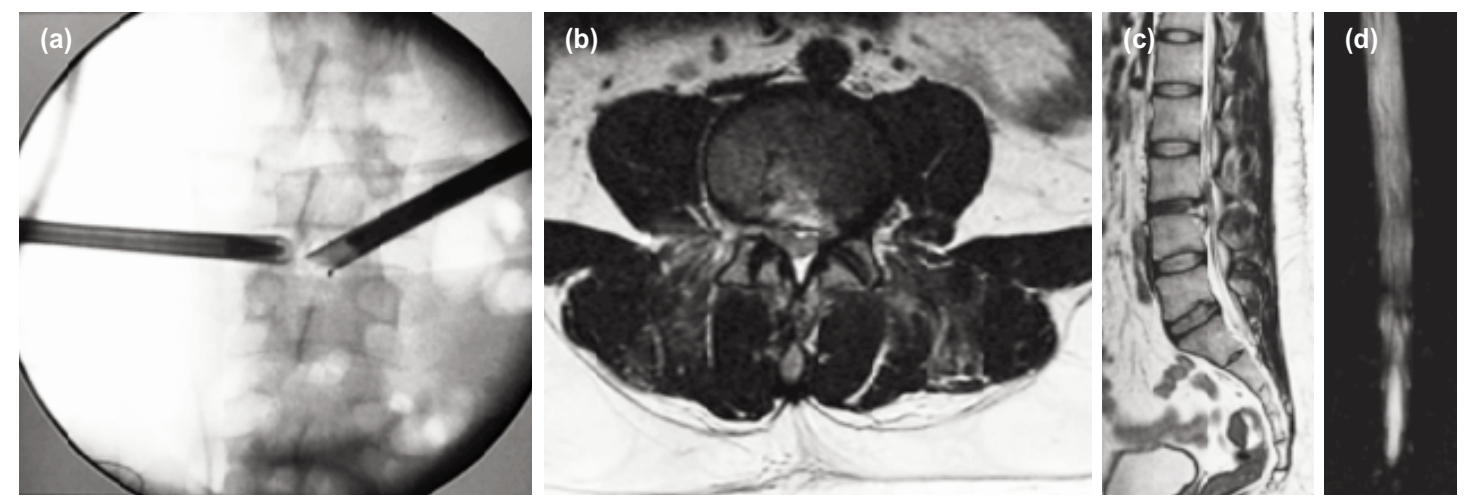

Fig. 4: (a) "Inside out" Bi-lateral Bi-portal approach taken for surgery. (b,c) Post-operative T 2 axial and saggital MRI showing complete decompression. (d) MR myelogram shows blockage cleared and the patient recovered within one day.

The medical comorbidities are not a contraindication for PTELD under $L^{8,11}$. In fact, the negativities of GA on the patient's visceral function (POUR-post-operative urinary retention) and cognitive dysfunction are not hidden and may have some critical role in immediate post-operative period in spine surgeries ${ }^{34}$. One patient of our series was operated under GA and he took one week to recover his bladder function. So, this aspect is completely avoided in CES patients operated under LA. Anaesthesiologist presence is mandatory to cover any emergency as well as to provide better communication.

However, there are negatives to PTELD and medico-legal implications of CES that need to be considered. Following PTELD post-operative, residual shadows may appear like pseudo-hernia. Lack of complete professional knowledge of competing surgeons and reporting by radiologists can cause disputes, especially if the patient has got residual or no recovery ${ }^{10,11}$. Another negative aspect is radiation exposure $^{35,36}$.

Dural tears, exiting nerve injuries, and dysesthesia can also occur in PTELD ${ }^{37}$. Our results are equally better as compared with other PTELD short series studies on objective parameters and the speed of complete recovery (Table III). The statistically significant improved ODI, VAS and patient satisfaction index are remarkable. Though no NILBP was noted in any patient, there were nine patients with ILBP. This necessitates that mandatory lifestyle modification needs to be followed by all patients.

In 13 out of 15 cases, basic techniques of surgery worked and BLBP approach was needed in two of our cases (Fig. 3, 4). This additional approach was also identified in one of the previous studies ${ }^{11}$. Rajasekaran et al suggest a conceptual change to understand that the disc prolapsed material can have associated endplate cartilage or/ and annulus in addition to nucleus fragment ${ }^{38}$. These fragments may be collagenized or calcified (CC) and must be removed to achieve complete decompression as was done in three of our cases.

There are many limitations to our study by its retrospective methodology itself. The study was a non-consecutive series. It is possible that less affected patients got enrolled in our present study even though our patients fitted into classic Fraser et al description of CES, and we followed Gleave et al classification of CESR and CESI ${ }^{4,23}$. The patient feedback for bladder dysfunction was purely subjective, and no supportive urodynamic studies were conducted to document and compare. However, it is also well established that patients may be asymptomatic despite the severe disruption of bladder function in urodynamic studies ${ }^{3,39}$. Moreover, in studies of surgery for CES, there exists a significant heterogeneity of reported outcomes ${ }^{1-4,22}$. This indicates a clear need for the development of a Core Outcome Set (COS), which has been suggested with the involvement of patient inputs, for uniformity of database ${ }^{22}$.

\section{CONCLUSION}

The superiority and curative outcome of the PTELD in CES is visible but more studies are needed before it is recommended for all cases due to the medico-legally volatile nature of the disabling condition.

\section{CONFLICT OF INTEREST}

The authors declare no conflicts of interest. 


\section{REFERENCES}

1. Gitelman A, Hishmeh S, Morelli BN, Joseph SA, Casden A, Kuflik P, et al. Cauda equina syndrome: a comprehensive review. Am J Orthop (Belle Mead NJ). 2008; 37(11): 556-62.

2. Kapetanakis S, Chaniotakis C, Kazakos C, Papathanasiou JV. Cauda equina syndrome due to lumbar disc herniation: a review of literature. Folia Med (Plovdiv). 2017; 59(4): 377-86. doi: 10.1515/folmed-2017-0038

3. Gardner A, Gardner E, Morley T. Cauda equina syndrome: a review of the current clinical and medico-legal position. Eur Spine J. 2011; 20(5): 690-7. doi: 10.1007/s00586-010-1668-3

4. Fraser S, Roberts L, Murphy E. Cauda equina syndrome: a literature review of its definition and clinical presentation. Arch Phys Med Rehabil. 2009; 90(11): 1964-8. doi: 10.1016/j.apmr.2009.03.021

5. Olivero WC, Wang H, Hanigan WC, Henderson JP, Tracey PT, Elwood PW, et al. Cauda equina syndrome (CES) from lumbar disc herniations. J Spinal Discord Tech. 2009; 22(3): 202-6. doi: 10.1097/BSD.0b013e31817baad8

6. Dave BR, Samal P, Sangvi R, Degulmadi D, Patel D, Krishnan A. Does the Surgical Timing and Decompression Alone or Fusion Surgery in Lumbar Stenosis Influence Outcome in Cauda Equina Syndrome?. Asian Spine J. 2019; 13(2): 198-209. doi: 10.31616/asj.2018.0168

7. Kim HS, Kim SW, Lee SM, Shin H. Endoscopic discectomy for the cauda equina syndrome during third trimester of pregnancy. J Korean Neurosurg Soc. 2007; 42(5): 419-20. doi: 10.3340/jkns.2007.42.5.419

8. Kim HS, Adsul N, Ju YS, Kim KJ, Choi SH, Kim JH, et al. Percutaneous Endoscopic Transforaminal Lumbar Discectomy for Massive Disc Herniation Causing Cauda Equina Syndrome: A Case Report. J Minim Invasive Spine Surg Tech. 2018; 3(1): 279. doi: https://doi.org/10.21182/jmisst.2017.00248

9. Jha SC, Tonogai I, Takata Y, Sakai T, Higashino K, Matsuura T, et al. Percutaneous endoscopic lumbar discectomy for a huge herniated disc causing acute cauda equina syndrome: A case report. J Med Invest. 2015; 62(1-2): 100-2. doi: 10.2152/jmi.62.100

10. Li X, Dou Q, Hu S, Liu J, Kong Q, Zeng J, et al. Treatment of cauda equina syndrome caused by lumbar disc herniation with percutaneous endoscopic lumbar discectomy. Acta Neurol Belg. 2016; 116(2):185-90. doi: 10.1007/s13760-015-0530-0

11. Namboothiri S, Gore S, Raja P. Novel Surgical Technique for discogenic cauda equina syndrome-transforaminal intra discal access by annulotomy outside central spinal canal. J Spine. 2016; S7. doi: 10.4172/2165-7939.S7-008

12. Mahesha K. Percutaneous endoscopic lumbar discectomy: Results of first 100 cases. Indian J Orthop. 2017; 51(1): 36-42. doi: 10.4103/0019-5413.197520

13. Gu YT, Cui Z, Shao HW, Ye Y, Gu AQ. Percutaneous transforaminal endoscopic surgery (PTES) for symptomatic lumbar disc herniation: a surgical technique, outcome, and complications in 209 consecutive cases. J Orthop Surg Res. 2017;12(1):25. doi: 10.1186/s13018-017-0524-0

14. Gore S, Yeung A. The "inside out" transforaminal technique to treat lumbar spinal pain in an awake and aware patient under local anesthesia: results and a review of the literature. Int J Spine Surg. 2014; 8: 28. doi: 10.14444/1028

15. Lewandrowsk KU. "Outside-in" technique, clinical results, and indications with transforaminal lumbar endoscopic surgery: a retrospective study on 220 patients on applied radiographic classification of foraminal spinal stenosis. Int J Spine Surg. 2014; 8: 26. doi: $10.14444 / 1026$

16. Ruetten S, Komp M, Godolias G. An extreme lateral access for the surgery of lumbar disc herniations inside the spinal canal using the full-endoscopic uniportal transforaminal approach-technique and prospective results of 463 patients. Spine (Phila Pa 1976). 2005; 30(22): 2570-8. doi: 10.1097/01.brs.0000186327.21435.cc

17. Tan M, Law LSC, Gan TJ. Optimizing pain management to facilitate enhanced recovery after surgery pathways. Can J Anaesth. 2015; 62(2): 203-18. doi: 10.1007/s12630-014-0275-x

18. Venkatesan M, Nasto L, Grevitt MP, Tsegaye MM. Is post-void bladder scan a useful adjunct to the clinical examination for prediction of cauda equine syndrome?. Spine J. 2017; 17(3): S7.

19. Cappelleri JC, Rosen RC. The Sexual Health Inventory for Men (SHIM): a 5-year review of research and clinical experience. Int J Impot Res. 2005; 17(4): 307-19. doi: 10.1038/sj.ijir.3901327 
20. Mixter WJ, Barr JS. Rupture of the intervertebral disc with involvement of the spinal canal. N Engl J Med. 1934; $211: 210-5$. doi: 10.1056/NEJM193408022110506

21. Fuso FAF, Dias ALN, Letaif OB, Cristante AF, Marcon RM, de Barros TEP. Epidemiological study of cauda equina syndrome. Acta Ortop Bras. 2013; 21(3): 159-62. doi: 10.1590/S1413-78522013000300006

22. Srikandarajah N, Wilby M, Clark S, Noble A, Williamson P, Marson T. Outcomes reported after surgery for cauda equina syndrome: a systematic literature review. Spine (Phila Pa 1976). 2018; 43(17): E1005-13. doi: 10.1097/BRS.0000000000002605

23. Gleave JRW, Macfarlane R. Cauda equina syndrome: what is the relationship between timing of surgery and outcome?. Br $J$ Neurosurg. 2002; 16(4): 325-8. doi: 10.1080/0268869021000032887

24. Tullberg T, Isacson J. Cauda equina syndrome with normal lumbar myelography. Acta Orthop Scand. 1989; 60(3): 265-7. doi: $10.3109 / 17453678909149274$

25. Bischoff RJ, Rodriguez RP, Gupta K, Righi A, Dalton JE, Whitecloud TS. A comparison of computed tomography-myelography, magnetic resonance imaging, and myelography in the diagnosis of herniated nucleus pulposus and spinal stenosis. $J$ Spinal Disord. 1993; 6(4): 289-95. doi: 10.1097/00002517-199306040-00002

26. Ahn UM, Ahn NU, Buchowski JM, Garrett ES, Sieber AN, Kostuik JP. Cauda equina syndrome secondary to lumbar disc herniation: a meta-analysis of surgical outcomes. Spine (Phila Pa 1976). 2000; 25(12): 1515-22. doi: 10.1097/00007632200006150-00010

27. Fehlings MG, Zeidman SM, Jha N, Rampersaud YR. Spine surgery -techniques, complication avoidance and management. In: Benzel EC, editor. Cauda equina syndrome. 3rd ed. Philadelphia: Elsevier/Saunders; 2012.

28. Podnar S. Cauda equina lesions as a complication of spinal surgery. Eur Spine J. 2010; 19(3): 451-7. doi: 10.1007/s00586-009$1170-\mathrm{y}$

29. Yeung AT, Tsou PM. Posterolateral endoscopic excision for lumbar disc herniation: surgical technique, outcome, and complications in 307 consecutive cases. Spine (Phila Pa 1976). 2002; 27(7): 722-31. doi: 10.1097/00007632-200204010-00009

30. Ruetten S, Komp M, Godolias G. Lumbar discectomy with the full- endoscopic interlaminar approach using new-developed optical systems and instruments. WSJ. 2006; 1(3):148-56.

31. Hellstrom P, Kortelainen P, Kontturi M. Late urodynamic findings after surgery for cauda equina syndrome caused by a prolapsed lumbar intervertebral disk. J Urol. 1986;135(2): 308-12. doi: 10.1016/s0022-5347(17)45621-7

32. Tan KA, Sewell MD, Markmann Y, Clarke AJ, Stokes OM, Chan D. Anterior lumbar discectomy and fusion for acute cauda equina syndrome caused by recurrent disc prolapse: report of 3 cases. J Neurosurg Spine. 2017; 27(4): 352-6. doi: 10.3171/2017.1.SPINE16352

33. Kim JS, Seong JH. Endoscope-assisted oblique lumbar interbody fusion for the treatment of cauda equina syndrome: a technical note. Eur Spine J. 2017;26(2): 397-403. doi: 10.1007/s00586-016-4902-9

34. Aiyer SN, Kumar A, Shetty AP, Kanna RM, Rajasekaran S. Factors influencing postoperative urinary retention following elective posterior lumbar spine surgery: a prospective study. Asian Spine J. 2018; 12(6): 1100-5. doi: 10.31616/asj.2018.12.6.1100

35. Webb J, Gottschalk IV L, Lee YP, Garfin S, Kim C. Surgeon perceptions of minimally invasive spine surgery. SAS J. 2008; 2(3): 145-9. doi: 10.1016/S1935-9810(08)70032-X

36. Choi KC, Lee JH, Kim JS, Sabal LA, Lee S, Kim H, et al. Unsuccessful percutaneous endoscopic lumbar discectomy: a singlecenter experience of 10,228 cases. Neurosurgery. 2015; 76(4): 372-380. doi: 10.1227/NEU.0000000000000628

37. Ahn Y, Lee HY, Lee SH, Lee JH. Dural tears in percutaneous endoscopic lumbar discectomy. Eur Spine J. 2011; 20(1): 58-64. doi: 10.1007/s00586-010-1493-8

38. Rajasekaran S, Bajaj N, Tubaki V, Kanna RM, Shetty AP. Anatomy of Failure in Lumbar Disc Herniation. An in Vivo, Multimodal, Prospective Study of 181 Subjects. Global Spine J. 2014; 4(1_suppl). doi: 10.1055/s-0034-1376749

39. Podnar S, Trsinar B, Vodusek DB. Bladder dysfunction in patients with cauda equina lesions. Neurourol Urodyn. 2006; 25(1): 23-31. doi: 10.1002/nau.20188 